\title{
X-RAY MICROSCOPY BY SUCCESSIVE FOURIER TRANSFORMATION *
}

\author{
J. T. WDNTHROP and C.R. WORTHINGTON \\ Department of Physics, University of Michigan, Ann Arbor, Michigan
}

Received 15 February 1965

$X$-radiation has a wavelength $\lambda$ of about $1 \AA$ and offers a higher resolution than light; the image resolution $\Delta r \approx \lambda / N A$. At the present time, the resolving power of the $\mathrm{X}$-ray microscope is no beiter than that of the light microscope, viz. $0.3 \mu$. We propose two new methods of X-ray microscopy which should provide improved resnlution; one method suggests a gain of acout 10 times. Both methods make use of two-wavelength microscopy; X-radiation is used to record the central portion of the Fourier transform of the object on film and optical reconstruction is accomplished by a lens. Experiments have not yet been performed to test the validity of the new methods; our proposal is based on theoretical considerations alone.

Coherent image formation by a lens makes use of two successive Fourier transformations. The Fourier transform of an object of transmittance $t(r)$ is denoted $T\left(r^{*}\right)$. A diffraction pattern $T\left(r^{*}\right)$ representing a central portion of the Fourier transform of the object, i.e. , finite $r^{*}$, is contained in the back focal plane of the lens. In general, the diffraction obtained without using a lens in Fresnel diffraction; the Fourier transform is a special case of a more general Fresnel transformation. Consider a simplified diffraction experiment. A point source is at a distance $p$ from the object plane $(r)$, and the amplitude $g\left(r_{1}\right)$ in the observation plane $\left(r_{1}\right)$, which is at a distance $q$ from the object plane, is given by the Fresnel-Kirchhoff relation. It is well-known that the amplitude is complex and the detector in the observation plane, i.e., a film, inly records intensities $\left|g\left(r_{1}\right)\right|^{2}$ so that the phases are lost.

In the Gabor light microscope (1), a coherent background wave is added and the resulting diffraction paitern is recorded on the film. This record (hologram) includes an interference term which contains the phases, and after optical processing (Fresnel transformation in this case) an

\footnotetext{
* This work was aided by U.S. Public Henlth Service Grant GM-09796.
}

image of the object results. The rrethod of Leith and Upatnieks (2) successfully removes the unwanted twin image. The Gabor microscope was originally proposed for two-wavelength operation using electrons and light, but the use of $X$-rays and light has often been considered (3-5). Our analysis (6) of the theoretical resolution of the Gabor X-ray microscope tends to confirm Baez (3) and it seems unlikely that this microscope would have higher resolution thin a lens.

Consider the operation of the Cabor microscope with $q$ sufficiently large so that the furfieid Fresnel condition is met (if the radius of the aperture is $R$ then the far-field condition is given by $R^{2} \ll 2 \lambda q$ ). The film records an interference term of the following form:

$$
\left|\eta^{\prime}\left(r^{*}\right)\right| \sin \left[\pi \frac{\lambda}{M} q\left(r^{*}\right)^{2}+\alpha(r)\right],
$$

where $T\left(r^{*}\right)=\left|T\left(r^{*}\right)\right| \exp \left[1 \alpha\left(r^{*}\right)\right], M$ is the shadow magnification and is given by $M=(p+q) / p$. The interference term has rapid vartation with increasing $r^{*}$ (which can be expressed in ternis of $r_{1}$ ) and therefore the far-field hologram falls to record the Fourier transform of the object apart from a tiny central portion. Hence the farfield Gabor X-ray microscope lacks resolution.

We propose two ways of recording the central portion of the Fourier transform of an object using $X$-radiation. Both methods have an interference term of the form

$$
\left|T\left(r^{*}\right)\right| \cos \left[2 \pi \beta \cdot r^{*}+\alpha\left(r^{*}\right)\right] \text {, }
$$

wher $\mathrm{\beta}$ is an experimental parameter. This intefex ence term can be recorded on film. Optical reconstruction using a lens then provides two magnified images of the object separated by a distance $2 M_{0} \beta$ where $M_{0}$ is the overall magnification.

Pinhole method. The source plane contains an $\mathrm{X}$-ray source of diameter $d$. The object plane is opaque and contains a small pinhole and a circular aperture of moderate radtus; these are separated by a center-to-center distance $\approx$. The 
object is placed over the circular aperture and the diffraction recorded on film. The experimental set-up is similar to a Young's interference system of two pinholes, (in order to get visible fringes the size of the source $d$ needs to be small, a condition which can easily be met). We note that this method also resembles that used by Leith and Upatnieks (7) for the production of Fourier holograms in light using a lens. $\mathrm{Pa}$ rallel may also be drawn with the heavy atom method of X-ray crystal structure analysis.

The transmittance for the object plane $t(r)$ is given by

$$
t(r)=t_{0}(r-a)+t_{1}(r),
$$

where the pinhole transmittance is $t_{0}(r-a)$ and the uniknown object is $t_{1}(r)$. The intensity recorded in the far-field is proportional to

$\left|T_{0}\left(r^{*}\right)\right|^{2}+\left|T_{1}\left(r^{*}\right)\right|^{2}+$

$+2\left|T_{0}\left(r^{*}\right)\right|\left|T_{1}\left(r^{*}\right)\right| \cos \left[2 \pi a \cdot r^{*}+\alpha_{1}\left(r^{*}\right)-\alpha_{0}\left(r^{*}\right)\right]$,

where

$$
T_{0}\left(r^{*}\right)=\left|T_{0}\left(r^{*}\right)\right| \exp \left[1 \alpha_{0}\left(r^{*}\right)\right]
$$

and

$$
T_{1}\left(r^{*}\right)=\left|T_{1}\left(r^{*}\right)\right| \exp \left[1 \alpha_{1}\left(r^{*}\right)\right] .
$$

The optical reconstruction using a lens has the autocorrelation functions of the pinhole and the object superimposed at the origin, and two cross correlations of the pinhole and the object located at $\pm M_{0} a$, i.e., a distance $2 M_{0} a$ apart.

The far-field condition for $\lambda \approx 1 \AA, q \approx 1$ meter requires $a \approx 3 \mu$ and it can be shown that the aperture size (radius) is about $2 \mu$. The resolution of the method $\Delta r \approx \delta$ where $\delta$ is the diameter of the pinhole; in order that $\Delta r \approx 500 \AA$ we need $\delta \approx 1 / \mu$. The small values of $\delta$ and $a$ are obvious limitations and present day exposure times would be enormous.

Esternal reflection method. If an $\mathrm{X}$-ray point source is placed in the object plane and an optical flat, l.e. , a mirror, is posttioned along $q$ so that external reflection takes place, then interference fringes are observed (6); this is the $X$-ray analog of Lloyd's mirror experiment in light.

The object plane is opaque and contains a circular aperture centered at $x=b$. The aperture has radius sllghtly less than $b$. An incoherent $X$-ray source is placed close to the aperture. A one-dimensional object $t(x)$ is placed over the aperturn. The intensity recorded contains the interference term

$$
\left|T\left(x^{*}\right)\right| \cos \left[2 \pi b \cdot x^{*}+\alpha\left(x^{*}\right)\right]
$$

where

$$
T\left(x^{*}\right)=\left|T\left(x^{*}\right)\right| \exp \left[1 \alpha\left(x^{*}\right)\right] \text {. }
$$

However, only a half-plane recording is obtained, i.e. , $x^{*} \geqslant 0$; a second copy is required before obtaining the optical reconstruction. The optical reconstruction contains a central peak and two magnified images of the object at $\pm M_{0} b$.

The resolution is given by $\Delta x=\lambda / 2 \theta_{c}$, where $\theta_{c}$ is the maximum angle of external reflection. The resolution is independent of wavelength and depends only on the material used for the mirror; a value of $\Delta x \approx 150 \AA$ is obtained using experimental values of $\theta_{c}$ and $\lambda(9)$.

Film resolving power and an experimental set-up of $\lambda \approx 1 \AA$ and $q \approx 1$ meter restrict the aperture size to about $50 \mu$.

Comments. The pinhole method is directly applicable to plane objects but information on the third dimension is preserved (the analysis is omitted). The external reflection method can be extended to two dimensions by use of two mirrors at right angles, but the third dimension is not preserved.

The overall magnification $M_{0}$ in the pinhole method is given by $M_{0}=\lambda_{2} q_{2} / \lambda_{1} q_{1}\left(\lambda_{1}, q_{1}\right.$ refers to the first stage using $X$-radiation; $\lambda_{2}, q_{2}$ refers to the optical reconstruction), which is identical to that of the two-wavelength Gabor microscope (1). The magnification in the external reflection method is given by $M_{0}=2 \lambda_{2} q_{2} / \lambda_{1} q_{1}$

The $X$-ray source size in the pinhole method has not been neglected; a finite incoherent source gpreads the intensity pattern from a point source by a convolution operation. The result of this effect is not to limit resolution, but to restrict the object dimension (6).

In the external reflecting method there will be some scattering trom the mirror surface (10). It is problematical to what extent the resolution will be affected. Preliminary considerations suggest that the resolution will be little changed but, more correctly, this can only be answered by experime.t. We note that the method has some advantages in comparison to the $X$-ray reflection method. It avoids the troublesome aberrations, has a higher magnification, a larger aperture stze, and uses shorter exposure times.

\section{References}

1. D.Gabor, Proc.Roy.Soc. (London) A 197 (1949) 454.

2. F.N. Leith and J. Upatnieks, J.Opt.Soc. Am. 52 (1962) 1123. 
3. A.V.Brez, J.Opt.Soc. Am. 42 (1952) 756.

4. B.N. Leith, private communication (1962).

5. G. W. Stroke and D.G. Falconer, Physics Letters 13 (1964) 306.

6. J.T. Winthrop and C.R. Worthington, in preparation.
7. E.J. Leith and J. Upatnieks, J.Opt.Soc. Am. 54 (1964) 1295.

8. G, Kellstrom, Nova Acta Soc.Scl. Upsal. 8 (1982) 61.

8. L, M.Rieser, J.Opt.Soc.Am, 47 (1957) 987.

10. W. Ehrenberg, J.Opt. Soc. Am. 39 (1949) 746.

\title{
ON THE SUSCEPTIBILITY OF LIQUID GADOLINIUM
}

\author{
R.M. XAVIBR, X.A. DA SILVA and W. BALTENSPERGER \\ Centro Brasileiro de Pesquisas Fistcas, Rio de Janeiro, Brasil
}

Recelved 9 February 1965

In the rare earth metals the ionic magnetic moments are coupled via the conduction electrons. This has often been studied for the soltd state [1-4]. In this work the effects of a liquid structure und of a short mean free path are evaluated. Although precise empirical data are lacking, the results clearly show that at high temperatures the shirt mean free path drastically decreases the paramagnetic Curie temperature, which, however, is larger in the liquid compared to the solid state.

The coupling between two ion spins has the form

$$
I_{i j}=\left(9 \pi Z^{2} \Gamma^{2 / 4 \mu}\right)(g-1)^{2} F_{\lambda}\left(2 k_{m} R_{i j}\right) J_{i} J_{j}
$$

where $Z$ is the ralency, $-\frac{3}{2} \Gamma$ the exchange integral between a if and a conduction electron, $g$ the Lande factor, $(g-1) J_{i}$ the component of the spin directed along the angular momentum of the ion $i[1]$, and $k m$ the wave number corresponding to the Fermi energy $\mu$. For an infinite mean free path $\lambda, F_{\infty}(x)=x^{-4}(x \cos x-\sin x)$ is the Ruderman-Kittel function. When $\lambda$ is finite, $F_{\lambda}$ is the integral expression given by Kaplan (ref. 5 equation 19 multiplied by $\left.\left(4 \pi k_{m}^{4} k^{2}\right)^{-1}\right)$.

In solids the total interaction energy is a sum of (1) over all lattice positions. In a liquid this energy is obtained by integrating (1) over the radial distribution function. Thus in the presence of a magnetic induction $B$ along the $z$ direction, the energy per ion in the paramagnetic region becomes

$E=-g i j B\left(J_{z i}+\left(9 \pi Z^{2} \Gamma^{2} / 8 \mu\right)(g-1)^{2}\left(J_{z}\right)^{2} S\right.$

where $\mu_{\mathrm{b}}$ is the Bohr magneton, and $(.$.$) the$ statistical average. The structure factor is

$$
\begin{gathered}
S=\sum_{j \neq 0} F_{\lambda}\left(2 k_{m} R_{0 j}\right) \\
S=\int_{0}^{\infty} 4 \pi r^{2} \rho(r) F_{\lambda}\left(2 k_{m} r\right) \mathrm{d} r
\end{gathered}
$$

for liquids, $\rho(r)$ being the atomic density at a distance $r$ from an ion. The paramagntic Curle temperature in the Welss approximation becomes

$$
T_{\mathrm{c}}=-3 \pi \Gamma^{2} Z^{2} S(g-1)^{2} J(J+1) /\left(4 k_{\mathrm{B}} \mu\right)
$$

( $k_{\mathrm{B}}$ is the Boltzmann constant).

$\rho(r)$ can be determined by $X$-ray or neutron diffraction. For liquid rare earths, however, these data are not available. We shall construct a. distribution function with the assumption that two elements with the same crystal structure at high temperatures have gimilar distribution functions in the liquid state. Thus, given $4 \pi r^{2} \rho_{A}(r)$ the radial distribution function for a liquid $A$, and a scale factor $\left.\alpha=\left(\rho_{O A} / \rho_{O B}\right)\right\}$ where $\rho_{0}$ is the atomic density, the proposed distribution in the liquid $B$ is $4 \pi(\alpha r)^{2} \alpha^{-3} \rho_{A}(\alpha r)$. As a test the radial distributions proposed for $\mathrm{Cs}$ based on the data of $\mathrm{Li}, \mathrm{Na}, \mathrm{K}$ and $\mathrm{Rb}[6]$ agree with the empirical distribution better than 4 and $0 \%$ regarding the positions of the first and second peaks, respectively, while the heights show a disagreement of $18 \%$ in the worst case. Since Gd has a b.c.c. structure at high temperatures [7], we use the data of $\mathrm{Cs}$.

The mean free path varies slowly at high temperatures. A value of $\lambda=5 \AA$ has been quoted for the solid phase [4]. Kaplan's expression $F_{\lambda}\left(2 k_{m} R\right)$ has been evaluated with an electronic computer.

The structure factors $S$ is an invariant of the 